\title{
A case for the resuscitation of infants born at less than 24 weeks gestation
}

\author{
John M. Dagle, MD, $\mathrm{PhD}^{1}$
}

Despite improvements in survival of extremely preterm infants, the decision whether to begin intensive care for infants at borderline viability is controversial. A great deal of this controversy focuses on concerns of survival, cost and neurodevelopmental outcome. Why are these concerns more emphasized in discussions of prematurity than other areas of medicine? In other words, why do we feel less obligated to treat extremely premature infants than older individuals in similar high risk situations? As more NICUs publish their experiences with infants in the 22-24 week gestational age range, we find survival statistics that cannot be labeled as futile if we are to be consistent with other patient populations. With respect to cost, NICU dollars are directed toward survivors at all birth weight categories and the percent of bed-days for non-survivors is much higher in adult ICUs compared to NICUs. The annual cost of caring for preterm infants in NICUs in the US is comparable to annual expenditures for either pet food or video games in our country, thus "scarcity of resources" arguments for limiting resuscitation of extremely preterm infants are not logical. With respect to quality of life, there are many reports supporting increased rates of neurodevelopmental impairment in extremely preterm infants. While these observations cannot be denied, it must be understood that an ICU admission at any age is a high-risk medical situation that can result in significant long-term sequelae. A stroke victim may have permanent neurocognitive issues, but they are admitted for evaluation. The medical community rightfully resuscitates infants with Down syndrome, and treats their medical issues, even though they are at high risk for cognitive impairments. Why is the situation different for 22-24 week preterm infants? Drawing lines with respect to who gets resuscitated is an easy way out of a decision that should not be easy. An assigned gestational age is often a "best guess" within a range of normal biologic variability governed by genetics and the environment. Perhaps we should let each infant make the decision regarding "viability". If a preterm infant is resuscitated, but soon succumbs to unresponsive respiratory failure, then we can discuss previabilty in retrospect. Designating an infant as previable based only on gestational age and making this decision before the child is born is similar to leaving an accident victim at the scene because they might be very badly injured. Why are extremely preterm infants treated substantially differently than other critically ill patients in many hospitals? A parent who desires a perfect child may request withholding support of a high-risk infant. Perhaps a lack of "personhood" plays a role in withholding care in that these infants are not communicative and have not yet developed a personality. Since abortions can be performed in some states at the gestational ages under discussion here, perhaps a diminished sense of responsibility underlies some of the decisions made regarding infants at the borderline of viability. Perhaps it is time we view prematurity in the same light as other medical conditions.

${ }^{1}$ Department of Pediatrics, The University of lowa, lowa City, IA, 52240

Please cite this paper as: Dagle JM. A case for the resuscitation of infants born at less than 24 weeks gestation. Proc Obstet Gynecol. 2011 November;2(2):Article 5 [ 1 p.]. Available from: http://ir.uiowa.edu/pog/. Free full text article.

Corresponding author: John M. Dagle, Department of Pediatrics, University of lowa, 8805 JPP, 200 Hawkins Drive, lowa City, IA, 52242. Telephone (319)353-7009, john-dagle@uiowa.edu

This is an Open Access article distributed under the terms of the Creative Commons Attribution 3.0 Unported License (http://creativecommons.org/licenses/by/3.0), which permits unrestricted use, distribution, and reproduction in any medium, provided the original work is properly cited. 